\title{
Fluorescence des gaz rares à haute pression excités par flash $\mathbf{X}$ rapide
}

C. Cachoncinlle, E. Robert, A. Khacef* et J.M. Pouvesle

GREMI, CNRS/Université d'Orléans, BP. 6759, 45067 Orléans cedex 2, France

* LPMI, Institut de Physique, USTHB, BP. 32, El Alia, Alger, Algérie

\begin{abstract}
In this work, we report on flash X-ray excitation of high pressure pure rare gases ( $\mathrm{He}, \mathrm{Ne}, \mathrm{Ar}, \mathrm{Kr}$, and $\mathrm{Xe}$ ) and mixture of rare gases. It is shown that high efficiency in energy deposition could be achieved using this new excitation means. Such an energetic excitation leads to important population of highly excited ionic species. As a consequence, the fluorescence emitted from the rare gases is mainly located in the UV-VUV wavelength domain. The high level of fluorescence observed authorized time resolved spectroscopy using conventional means. The dominant feature of the spectra appears to be the second and third continua of rare gases. The use of this specific technics of excitation leads to an efficient production of heteronuclear ions at high pressure.
\end{abstract}

\section{INTRODUCTION.}

La recherche de nouvelles sources de photons dans le domaine spectral des photons UV-X amène tout naturellement au développement de nouvelles techniques de dépôt d'énergie dans les états très excités des constituants de la matière. Bien souvent, ces techniques sont relativement sophistiquées (rayonnement synchrotron, faisceau d'ions,...) et non accessibles au niveau d'un laboratoire. Depuis quelques années, nous avons choisi d'étudier la fluorescence UV-VUV issue de la photoionisation des gaz rares à haute pression (jusqu'à 30 bars) par des photons $X$ d'énergie comprise entre quelques keV et quelques dizaines de $\mathrm{keV}$ produits par un flash $\mathrm{X}$ de forte puissance crête (quelques centaines de $\mathrm{kW}$ ) développé spécialement pour ce type d'étude. Nous allons présenter ici les grandes lignes de ce travail.

\section{DISPOSITIF EXPERIMENTAL.}

Le dispositif expérimental est présenté figure 1 . Il se compose principalement de trois parties: le système d'excitation des gaz par impulsions de photons X (ou "flash X") 


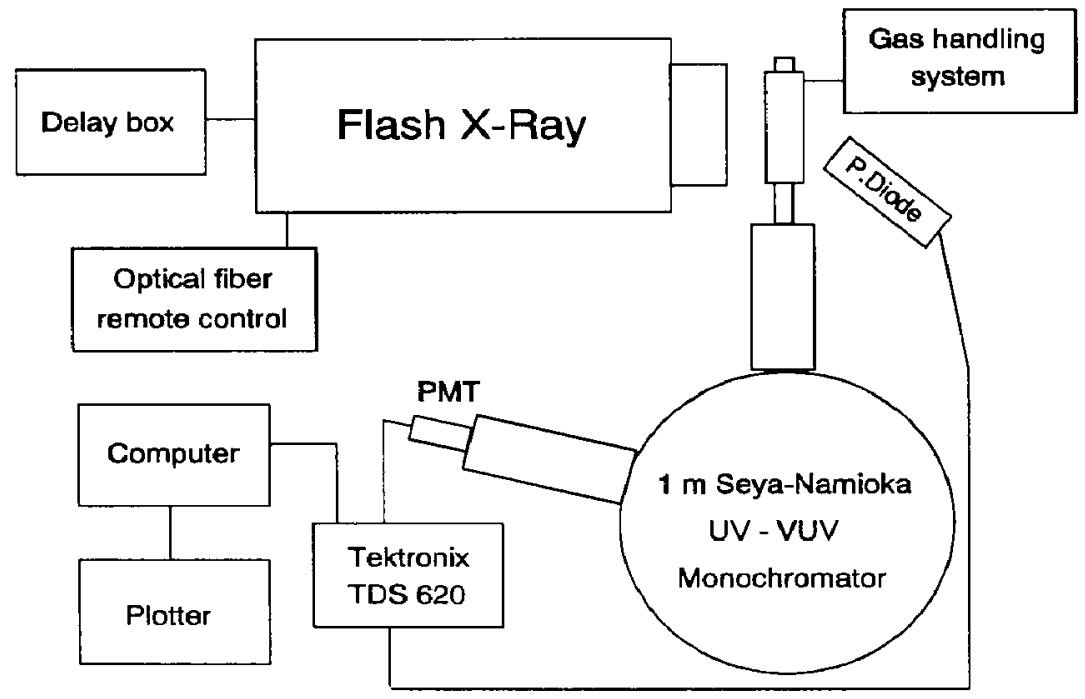

Figure 1.: Dispositif expérimental.

accompagné de son électronique de déclenchement, l'ensemble de gestion des gaz à des pressions multi-atmosphériques comprenant une cellule d'étude de la fluorescence émise par le gaz et, enfin, une chaîne d'acquisition des données permettant les études spectroscopiques résolues en temps.

Le flash $\mathrm{X}$ utilisé a été spécialement conçu pour la photoionisation de gaz et mélanges de gaz à très hautes pressions. Il permet de générer des impulsions de photons $X$ très intenses et de courte durée. Ce système bâti autour d'un concept développé par C.B. Collins et al [1] se caractérise par sa très grande souplesse d'utilisation et son faible encombrement $\left(1.4 \times 0.3 \times 0.3 \mathrm{~m}^{3}\right)$. La présentation détaillée de ses caractéristiques et l'étude de ses performances ont déjà fait l'objet d'une précédente publication [2]. Nous ne donnons ici qu'un simple rappel de ses principales caractéristiques.

La source de photons $\mathrm{X}$ a la forme d'une lame de $10 \mathrm{~cm}$ de long et environ $1 \mathrm{~mm}$ de hauteur. La géométrie rectangulaire du faisceau permet un éclairement homogène du gaz dans la cellule d'étude. Le spectre en énergie des photons $X$ émis par le générateur s'étend de 5 à $40 \mathrm{keV}$. Il est constitué d'un fond de rayonnement continu, dû au freinage des électrons ("bremsstrahlung"), auquel se superpose le spectre des raies caractéristiques du matériau constituant l'anode (Cu, Mo ou W) [3].

Les impulsions ont une durée qui peut être ajustée entre quelques nanosecondes et quelques dizaines de nanosecondes. La dose par tir mesurée à la fenêtre de sortie peut atteindre 2 rads pour une énergie stockée maximale de $6 \mathrm{~J}$. Le nombre de photons émis à chaque tir correspond à un flux de l'ordre de quelques $10^{10}$ photons par centimètre carré. Au niveau de la fenêtre d'entrée de la cellule, le dépôt d'énergie dans le gaz peut atteindre quelques centaines de microjoules. Le flash peut fonctionner jusqu'à $50 \mathrm{~Hz}$, valeur supérieure à la limite imposée par la chaîne d'acquisition pour les études de spectroscopie résolue en temps. L'ensemble des données présenté dans ce papier a été obtenu à une 
fréquence de répétition comprise entre 10 et $20 \mathrm{~Hz}$. A cette cadence de tir, le flux de photons délivré par la source peut être maintenu constant pendant plusieurs heures.

Le système de gestion des gaz permet de travailler en écoulement (200 à 2000 $\mathrm{cc} / \mathrm{min}$ ) dans une gamme de pression allant de 0 à 30 bars. Lorsque la cellule n'est pas utilisée, elle est maintenue sous vide secondaire par un groupe de pompage turbomoléculaire.

La cellule d'étude contenant le gaz à haute pression est de géométrie rectangulaire $\left(10 \times 2 \times 1 \mathrm{~cm}^{3}\right)$. L'excitation du gaz se fait transversalement alors que l'observation de la fluorescence émise par le gaz se fait longitudinalement. Les photons $X$ pénètrent dans la cellule au travers d'une fenêtre constituée d'une fine feuille de Kapton $(50 \mu \mathrm{m})$. Cette feuille est du même type que celle qui équipe la fenêtre de sortie du flash; elle est transparente aux photons d'énergie supérieure à $5 \mathrm{keV}$. Les excellentes qualités mécaniques du Kapton permettent d'atteindre dans la cellule des pressions de 30 bars. La cellule est pourvue de hublots en $\mathrm{MgF}_{2}$ suivant son axe longitudinal. Ce matériau est transparent dans l'ultraviolet du vide (VUV) jusqu aux environs de $110 \mathrm{~nm}$. La cellule est connectée à un spectromètre VUV par un tube de transfert. Ces deux derniers éléments sont maintenus en permanence sous vide secondaire.

Le spectromètre (Seya-Namioka, $1 \mathrm{~m}$ ) est couplé à une chaîne d'acquisition de données adaptée aux études de spectroscopie résolue en temps. Il est équipé d'un jeu de deux réseaux et de tubes photomultiplicateurs interchangeables permettant de couvrir le domaine spectral qui s'étend du VUV $(110 \mathrm{~nm})$ au proche infrarouge $(800 \mathrm{~nm})$. Ce système possède un pouvoir de résolution spectrale relativement faible (Résolvance de 100 à $200 \mathrm{~nm}$ ). Les tubes photomultiplicateurs (Hamamatsu R955 et R1080) ont des temps de réponse de l'ordre de quelques nanosecondes. Le signal issu de ces détecteurs est envoyé sur un oscilloscope numérique (Tektronix TDS 620) utilisé comme analyseur de transitoire et interfacé avec un microordinateur pour le stockage et le traîtement des données. Les données sont considérées comme une surface à deux dimensions (longueur d'onde et temps). Une coupe dans cette surface à longueur d'onde donnée fournit l'évolution temporelle de la fluorescence à cette longueur d'onde, alors qu'une coupe à un temps donné permet d'obtenir le spectre d'émission à un certain moment de l'histoire du plasma. La résolution temporelle du système est de 2 ns. La chaîne d'acquisition est synchronisée sur l'impulsion de photons $X$ au moyen d'une photodiode rapide sensible aux photons d'énergie de plus de $5 \mathrm{keV}$ conduisant à un "jitter" de l'ordre de $2 \mathrm{~ns}$.

\section{DEPOT D'ENERGIE.}

Dans le domaine d'énergie des photons $X$ allant de 5 à $50 \mathrm{keV}$, l'interaction d'un photon $\mathrm{X}$ avec un atome conduit à la création d'une paire électron-ion. La probabilité de cet événement est par définition proportionnelle à la section efficace, $\sigma$, du processus considéré. Pour ce type d'interaction, les valeurs des sections efficaces sont parfaitement connues et tabulées pour tous les gaz rares. Elles dépendent de l'énergie $E$ des photons incidents. On notera $\sigma(E)$ cette section efficace. L'énergie alors transférée au gaz, sous forme d'ionisation de ses constituants, est directement proportionnelle au flux de photons $X$ local: c'est la loi de Beer-Lambert:

$$
I(x)=I_{0} e^{-\sigma(E) N x}
$$

où $\mathrm{N}$ est la densité du gaz et $\mathrm{x}$ la distance parcourue dans le milieu. 
Pour une cellule de profondeur L, l'énergie déposée dans le milieu gazeux est donc fonction de sa densité (reliée à la pression du gaz) et suit alors la loi:

$$
E_{d}=E_{0}\left(1-e^{-\sigma(E) N L}\right)
$$

où $E_{0}$ est l'énergie transportée par le faisceau incident et $E_{d}$ est l'énergie déposée dans la cellule d'étude. L'efficacité du processus est très dépendante de la nature du gaz. Puisque les sections efficaces augmentent avec la taille de l'atome (donc avec sa masse atomique), le processus, qui dépend exponentiellement de cette section efficace, sera d'autant plus probable que le gaz rare utilisé aura une masse atomique importante. Si nous pouvons déposer pratiquement $100 \%$ de l'énergie transportée par le faisceau de photons $\mathrm{X}$ dans du xénon dès des pressions de quelques bars, il est par contre impossible d'obtenir un dépôt d'énergie significatif dans de l'hélium pour des pressions inférieures à 50 bars.

La photoionisation du gaz sous l'impact d'un photon de grande énergie résulte principalement de l'éjection d'un électron d'une des couches les plus internes de l'atome. En fait, si l'énergie du photon $X$ est suffisante, l'éjection se fera à partir de la couche dont les électrons possèdent l'énergie de liaison la plus résonnante avec l'énergie du photon incident. Ainsi, des photons de $10 \mathrm{keV}$ vont provoquer l'ëjection d'un électron des couches $\mathrm{K}$ du néon et de l'argon (seuil à $0.87 \mathrm{keV}$ et $3.2 \mathrm{keV}$ respectivement) mais ils n'auront pas l'énergie suffisante pour créer un trou dans les couches $\mathrm{K}$ du krypton et du xénon (situées à $14.3 \mathrm{keV}$ et $34.6 \mathrm{keV}$ respectivement). Pour ces deux derniers gaz, lionisation de l'atome se produira par éjection d'un électron d'une des couches $L$ d'ènergie de liaison plus faible.

Ce processus de photoionisation des couches internes constitue le mécanisme primaire de dépôt d'énergie dans le milieu, mais n'est pas le mécanisme prépondérant conduisant à la génération d'états hautement excités. En effet, l'éjection d'un des électrons internes du cortège électronique conduit à l'éjection d'autres électrons de l'atome à la suite de processus en cascade résultant du réarrangement des différentes couches électroniques. Aussi, l'interaction d'un seul photon $X$ énergétique conduit à la formation d'un ion de gaz rare $(R g)$ plusieurs fois ionisé: $\operatorname{Rg}^{n+}(n>1)$. L'abondance relative des différents ions multichargés est bien sûr fonction de la nature du gaz et de l'énergie des photons incidents. Ceci est parfaitement bien connu [4]. A titre d'exemple, l'ionisation de l'argon par des photons de $10 \mathrm{keV}$ conduit principalement à la formation d'ions $\mathrm{Ar}^{4+}(40 \%)$ et $\mathrm{Ar}^{5+}$ $(30 \%)$, l'espèce ionique une seule fois ionisée étant nettement minoritaire.

Il serait pourtant erroné de croire que, lors de l'excitation d'un gaz rare par flash $\mathrm{X}$, les ions multichargés constituent l'espèce ionique dominante. En effet, les n électrons très énergétiques éjectés des couches internes vont à leur tour effectuer des collisions avec les atomes environnants. L'énergie de ces électrons étant supérieure au seuil d'ionisation du gaz rare, la probabilité de création d'une nouvelle paire ion-électron est très importante. Les sections efficaces d'ionisation par collision avec des électrons rapides sont telles que l'interaction conduit plutôt à la formation de plusieurs ions une seule fois chargé $\mathrm{Rg}^{+}$qu'à la génération d'un seul ion plusieurs fois chargé $\mathrm{Rg}^{\mathrm{n}+}$. L'abondance relative de ces ions est dans le rapport $\sigma\left(\mathrm{Rg}^{\mathrm{n}}\right) / \sigma\left(\mathrm{Rg}^{+}\right)$des sections efficaces du processus dionisation par collision électronique. Ce rapport est fonction de la nature du gaz rare et il devient rapidement insignifiant pour $n>3$. Par exemple, pour des électrons incidents de $100 \mathrm{eV}$, le rapport $\sigma\left(\mathrm{Rg}^{2+}\right) / \sigma\left(\mathrm{Rg}^{+}\right)$varie entre 0.06 pour l'argon [5] et 0.12 [6] pour le xénon. Pour ces mêmes gaz le rapport $\sigma\left(\mathrm{Rg}^{3+}\right) / \sigma\left(\mathrm{Rg}^{+}\right)$tombe à des valeurs comprises entre 0.002 et 0.04 . Ainsi, si nous dressons un bilan des espèces ioniques créées par un unique photon $X$ 
de $10 \mathrm{keV}$, ces différents processus plus ou moins efficaces conduisent à la formation de quelques centaines d'ions une seule fois chargés, quelques dizaines d'ions deux fois chargés et seulement quelques ions portant une charge supérieure.

Ainsi, un flux de photons de quelques $10^{10}$ photons par centimètre carré conduit à une densité électronique moyenne de quelques $10^{12}$ électrons (ou ions) par centimètre cube au sein du gaz.

Il est important de noter que la loi de Beer-Lambert (1) presente une dépendance exponentielle en fonction de la profondeur $\mathrm{x}$ du milieu traversé. Il en résulte un dépôt d'énergie fortement inhomogène aux pressions les plus élevées. La densité électronique peut varier de plus d'un ordre de grandeur sur la largeur de la cellule. Alors que la densité électronique moyenne sur l'ensemble de la cellule $\left(10^{12}\right.$ électrons $\left./ \mathrm{cm}^{3}\right)$ est limitée par le flux de photons pénétrant dans la cellule $\left(10^{10}\right.$ photons $\left./ \mathrm{cm}^{2}\right)$, la très forte inhomogénéité du dépôt permet d'atteindre des densités électroniques locales de quelques $10^{14}$ électrons par centimètre cube si la densité du gaz est suffisante pour stopper le flux de photons incidents sur les premiers centaines de micromètres traversés.

La durée de vie du "plasma" est complètement dominée par les processus de recombinaison électron-ion. Pour les densités de gaz auxquelles nous travaillons (1-30 atmosphères à température ambiante), le temps de vie des ions monoatomiques $\mathrm{Rg}^{+}$est extrêmement court. Ces ions sont très rapidement transformés en dimères ioniques $\mathrm{Rg}_{2}{ }^{+}$ par des processus collisionnels à trois corps particulièrement efficaces. Pour fixer un ordre de grandeur, dans de l'argon à une pression de 10 bars, le temps de vie de $\mathrm{Ar}^{+}$est de l'ordre de la picoseconde. Ce processus de dimérisation des ions monoatomiques n'est qu'un cas particulier de processus collisionnels plus généraux aboutissant à la formation d'agrégats chargés, $\operatorname{Rg}_{\mathrm{m}}{ }^{\mathrm{n}}$.

Le diagnostic de la présence de telle ou telle espèce ionisée dans son état fondamental n'est pas trivial dans ces milieux à très hautes pressions. On peut, a priori, estimer que les agrégats $\mathrm{Rg}_{\mathrm{m}}{ }^{+}$sont présents car ces ions sont stables si leur taille n'est pas trop élevée. Par contre la présence d'agrégats $\mathrm{Rg}_{\mathrm{m}}{ }^{\mathrm{n}+}$ de charge $\mathrm{n}$ plus élevée est beaucoup moins certaine. Cela est dû phénomène d'explosion coulombienne qui provoque leur fragmentation. Dans nos conditions expérimentales, la formation de ces agrégats ioniques $\mathrm{n}$ fois chargés $(\mathrm{n}>1)$ résulte des forces attractives de polarisation entre un ion atomique $\mathrm{Rg} \mathrm{n}^{+}$et les atomes neutres environnants selon le schéma collisionnel [7] suivant

$$
\mathrm{Rg}^{++}+2 \mathrm{Rg} \rightarrow \mathrm{Rg}_{?}^{++*}+\mathrm{Rg}
$$

La formation de ces agrégats, dans leur état fondamental, à partir de collisions telles que

$$
\mathrm{Rg}^{\prime}+\mathrm{Rg}{ }^{\prime}+\mathrm{Rg} \rightarrow \mathrm{Rg}_{2}{ }^{\prime *}+\mathrm{Rg}
$$

est à proscrire car elle demanderait une énergie cinétique supplémentaire de quelques $\mathrm{eV}$ [8] pour vaincre la répulsion coulombienne. Par conséquent, seuls les états excités de ces ions ("excimères ioniques") $\mathrm{Rg}_{2}{ }^{\mathrm{n}+*}$ peuvent être formés. Dans le cas précis du dimère ionique $\operatorname{Rg}_{2}{ }^{2+*}$, la désexcitation a lieu par transfert de charge accompagné de l'émission d'un photon de grande énergie [7]. La formation d'excimères ioniques de ce type de plus grande taille $(m=3, \ldots)$ est peu probable. En effet les collisions du type

$$
\mathrm{Rg}^{++}+\mathrm{mRg} \rightarrow \mathrm{Rg}_{\mathrm{m}}^{++*}+\mathrm{Rg}
$$

peuvent conduire à l'explosion coulombienne vers des états situés à des niveaux d'énergie inférieure très proches tels que ceux corrélant vers $\mathrm{Rg}^{+}+\mathrm{Rg}^{+}+\mathrm{Rg}^{*}$ ou $\mathrm{Rg}_{2}{ }^{+*} \mathrm{Rg}^{+}$. Les 
surfaces de potentielles issues de ces états répulsifs vont prédissocier celles correspondant à l'excimère ionique de telle sorte que la stabilisation collisionnelle de l'agrégat $\operatorname{Rg}_{\mathrm{m}} \mathrm{n}^{\mathrm{n}^{*}}$ $(n>1, m>2)$ est très peu probable.

Aussi nous devons considérer que la photoionisation de gaz rares à haute pression par excitation $X$ conduit principalement à la production des agrégats ioniques dans leur état fondamental $\mathrm{Rg}^{+}$et excités $\mathrm{Rg}_{\mathrm{m}}{ }^{+*}$ (états de Rydberg) [9], mais aussi à la formation du dimère ionique $\mathrm{Rg}_{2}{ }^{2+*}$. La présence de plus grands agrégats d'ions multichargés est très peu probable. L'abondance relative de ces ions moléculaires est alors fonction de l'abondance relative des ions monoatomiques correspondants, soit, en ordre de grandeur, $90 \%$ d'ions $\mathrm{Rg}_{\mathrm{m}}{ }^{+}$(excités ou dans leur état fondamental) et $10 \%$ d'ions $\mathrm{Rg}_{2}{ }^{2+*}$.

\section{ANALYSE DE LA FLUORESCENCE.}

Nous avons vu que le dépôt d'énergie dans le gaz peut causer des gradients importants de la densité électronique. Ces inhomogénéités sont d'autant plus importantes que la densité des neutres est élevée et que la masse atomique du gaz considéré est grande. Aussi, l'analyse de la fluorescence du gaz doit prendre en considération ce gradient car la cinétique de certains processus est très dépendante de la densité électronique. Par exemple, la fréquence de recombinaison dissociative des ions moléculaires:

$$
\mathrm{Rg}_{2}^{+}+\mathrm{e}+\mathrm{e} \rightarrow \mathrm{Rg}^{*}+\mathrm{Rg}+\mathrm{e}
$$

dépend.du carré de cette densité. Par conséquent cette réaction peut parfaitement être importante dans une zone et complètement négligeable devant d'autres réactions dans une autre zone

L'analyse qualitative du spectre de la fluorescence émise entre 110 et $800 \mathrm{~nm}$ montre que l'énergie rayonnée est principalement émise dans le domaine spectral UV et VUV pour tous les gaz rares utilisés (voir fig. 2): argon, krypton et xénon. Notons que les essais effectués avec les gaz rares plus légers (hélium et néon) se sont révélés assez décevants. Aux pressions inférieures à 30 bars, l'hélium est beaucoup trop transparent aux photons $X$ délivrés par la source conduisant à un dépôt d'énergie négligeable dans le gaz. Si cette absence de fluorescence était prévisible pour l'hélium, on s'attend par contre à observer une fluorescence importante du néon dans la mesure où $80 \%$ de l'énergie est effectivement déposée dans ce gaz à 30 bars. En fait aucune fluorescence significative n'a pu être observée dans le domaine spectral couvert par le dispositif expérimental. La restitution de l'énergie se fait probablement à des longueurs d'onde inférieures à $110 \mathrm{~nm}$. Il est important de souligner que les transitions entre les différents états excités du néon $(550-750 \mathrm{~nm})$ sont restées invisibles.

Les spectres d'émission des trois gaz rares ( $\mathrm{Ar}, \mathrm{Kr}$ et $\mathrm{Xe}$ ) conduisant à une fluorescence importante présentent des similitudes. Ils font apparaître les structures bien connues des seconds continus, respectivement situés vers 125,145 et $175 \mathrm{~nm}$, et des troisièmes continus, vers 210,250 et $270 \mathrm{~mm}$. Les transitions à l'origine des seconds continus sont parfaitement identifiés depuis fort longtemps [10]. Ils correspondent à la désexcitation radiative des deux états moléculaires excités les plus bas en énergie de la molécule neutre $\mathrm{Rg}_{2}$. Par contre, la discussion reste très ouverte sur l'identification du ou des états moléculaires à l'origine des troisièmes continus des gaz rares [7-9,11-14] observés pour la première fois il y a trente ans. Il est cependant aujourd'hui acquis que ces continus sont d'origine ionique 


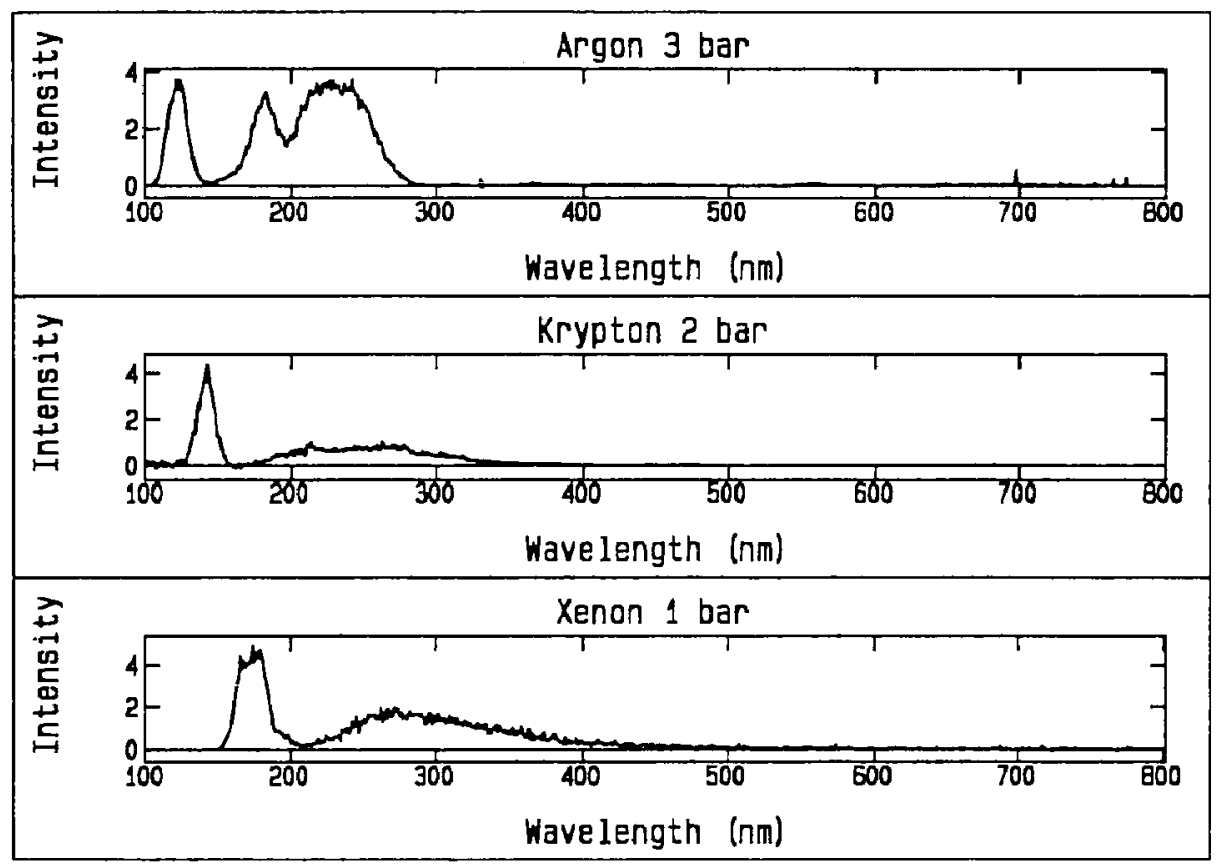

Figure 2: Fluorescence des gaz rares en excitation par flash $\mathrm{X}$.

L'observation des spectres d'argon à des pressions de quelques bars révèle la présence de quatre raies spectrales atomiques $(772.4 \mathrm{~nm}, 763.5 \mathrm{~nm}, 751.4 \mathrm{~nm}$ et 696.5 $\mathrm{nm}$ ) de faible intensite. Il est intéressant de noter que toutes les transitions observées proviennent d'un seul et mème groupe d'états excités $(\operatorname{Ar}(4 p))$ dont certains sont très spécifiquement peuplés par le processus de recombinaison dissociative à partir de l'ion moléculaire dans son état fondamental. Il apparait donc que les seuls états du neutre peuplés lors de l'excitation du gaz par flash $X$ le sont par recombinaison dissociative de l'ion moléculaire et non pas à l'issue de processus collisionnels impliquant des électrons énergétiques et des atomes neutres dans leur état fondamental. En d'autres termes, l'excitation de gaz rares par flash $X$ rapide conduit à déposer l'énergie pratiquement exclusivement dans des états ioniques. Cette dernière remarque singularise fortement l'excitation par flash X par rapport aux autres techniques d'excitation plus conventionnelles (excitation par faisceau d'électron, décharge rapide). Enfin, on peut justifier l'absence de fluorescence du néon dans le domaine spectral visible par le très faible peuplement des états excités de l'atome neutre dans ce type d'excitation par flash X.

Le dépôt effectif de l'énergie dans des états ioniques est mis en évidence sur les spectres enregistrés dans différents mélanges de gaz rares. Dans de tels mélanges, nous observons les bandes de fluorescence très significatives des excimères ioniques hétéronucléaires $\left(\mathrm{Rg}_{\mathrm{a}} \mathrm{Rg} \mathrm{g}^{+}\right.$(où les indices $\mathrm{a}$ et $\mathrm{b}$ référencent les deux gaz rares) des 
transitions de type $\left(\mathrm{Rg}_{\mathrm{a}} \mathrm{Rgb}_{\mathrm{b}}{ }^{+}\right)-\left(\mathrm{Rg}_{\mathrm{a}}{ }^{+} \mathrm{Rg}_{\mathrm{b}}\right)$ [15]. Ainsi, dans des mélanges à base d'argon (gaz majoritaire) nous avons observé les bandes de $(\mathrm{ArNe})^{+}$à $216 \mathrm{~nm}$ et $224 \mathrm{~nm}$ ainsi que celles de $(\mathrm{ArXe})^{+}$situées a 328 et $508 \mathrm{~nm}$. Pour ces exciplexes ioniques, l'état supérieur de la transition est corrélé à $\mathrm{Rg}_{\mathrm{a}}+\mathrm{Rg}_{b}{ }^{+}$où $\left(\mathrm{Rg}_{b}{ }^{+}\right)$est le plus léger des deux gaz rares (celui qui a le potentiel d'ionisation le plus élevé) alors que l'état inférieur de la transition est corrélé à $\left(\mathrm{Rg}_{b}+\mathrm{Rg}_{\mathrm{a}}{ }^{+}\right)$. Dans le cas du mélange argon-xénon, la formation de l'exciplexe résulte probablement de collisions entre l'ion du gaz majoritaire $\mathrm{Ar}^{+}$et des atomes du gaz minoritaire Xe. Par contre, le peuplement très significatif de l'état supérieur de l'ion (ArNe) ${ }^{+}$ne peut s'expliquer aussi simplement dans la mesure où le gaz qui possède le potentiel d'ionisation le plus élevé, $\mathrm{Ne}$, est le gaz minoritaire. L'ion $\mathrm{Ne}^{+}$est donc probablement extrêmement peu peuplé directement par excitation $\mathrm{X}$ du fait de sa très faible concentration partielle (1/1000). De plus, l'analyse des évolutions temporelles montre que la fluorescence de cette espèce atteint son maximum relativement tard comparée à l'impulsion d'excitation suggérant ainsi l'implication de plusieurs processus collisionnels aboutissant au peuplement de l'ion $\mathrm{Ne}^{+}$. De tels processus impliqueraient des états de l'argon, excités, ioniques, moléculaires ou atomiques et possédant une énergie supérieure à l'énergie d'ionisation du néon $(21.6 \mathrm{eV})$. On peut raisonnablement penser que les états de Rydberg de l'ion moléculaire d'argon $\mathrm{Ar}_{2}{ }^{+*}$ (à $\sim 26 \mathrm{eV}$ ) pourraient jouer un rôle dans ce processus de peuplement de $(\mathrm{ArNe})^{+}$.

\section{CONCLUSION.}

L'utilisation d'un flash $\mathrm{X}$ intense comme source de photons pour photoioniser un gaz à haute pression conduit principalement à la production de continus de fluorescence dans le domaine des photons UV et VUV. Nous avons montré ainsi que cette nouvelle technique d'excitation conduit au peuplement très significatif d'états ioniques. En particulier, la fluorescence émise par différents excimères ioniques a pu être observée à des pressions supérieures à la dizaine d'atmosphères.

Ce travail a bénéficié du soutient financier de la Région Centre.

\section{Références}

[1] C.B. Collins et al, Rev. Sci.Instrum. 57 (1986) 863.

[2] J.M. Pouvesle et al, Rev. Sci. Instrum. 64 (1993) 2320.

[3] F. Davanloo et al, in advances in laser science III edited by A.C.Tam (American Institute of Physics, New York, 1988), A.I.P. Conf.Proc. N'172, pp 62-64.

[4] T.A. Carlson et al, Phys. Rev, 151 (1966) 41.

[5] V.Tarnovsky et al, Z. Phys. D, 22 (1992) 603

[6] J. A. Syage, Phys. Rev. A, 46 (1992) 5666

[7] H. Langhoff, Opt. Commun., 68 (1988) 31

[8] C. Cachoncinlle et al., J. Chem. Phys, 96 (1992) 6085

[9] C. Cachoncinlle et al., J. Chem. Phys, 96 (1992) 6093

[10] Y Tanaka et al, J. Opt. Soc. Am., 48 (1958) 304

[11] G. Klein et al, J. Phys.B, 14 (1981) 1283.

[12] P. Millet, J. Phys. B, 15 (1982) 12935.

[13] T. D. Strickler, J. Chem. Phys, 41 (1964) 1783.

[14] G.S. Hurst, Phys. Rev., 178 (1969) 4.

[15] Y. Tanaka et al, J. Chem. Phys., 62 (1975) 4484 